\title{
Prevalence and Pattern of Complementary and Alternative Medicine for Autism Spectrum Disorder in Tamil Nadu
}

\author{
Udayakumar Narasimhan ${ }^{1}$ - Ramya Rajendran ${ }^{2}$ - Debi Ann Abraham ${ }^{2}$ - Lavanya Rajendran ${ }^{2}$. \\ Rajanandh Muhasaparur Ganesan ${ }^{2}$ (B)
}

Received: 18 September 2019 / Accepted: 21 November 2019 / Published online: 2 January 2020

(C) Dr. K C Chaudhuri Foundation 2020

To the Editor: Autism is a disorder, not a disease, and as such, there is still no formal treatment [1]. Complementary and alternative medicine (CAM) therapies are supplemental remedies, and should be combined with behavioral therapy as well, according to The Autism Society $[2,3]$. A survey was conducted among the parents/caregivers of autism spectrum disorder (ASD) children to estimate the prevalence and type of CAM use that they have used with their children. A total of 213 parents/caregivers of ASD children were interviewed about the type of CAM usage, recommendation source, reasons, expectations, effectiveness, harmful effects, if any and pediatrician's opinion on the usage of CAM. The data depict that the male gender is most affected by ASD in both CAM users $(80.5 \%)$ and non-CAM users $(68.6 \%)$ and children in the age range of 1 to 5 were mostly affected with ASD in both CAM users $(63.4 \%)$ and non-CAM users (75\%). Only $19.24 \%$ of the children were exposed to atleast one CAM therapy by their parents/caregivers. The most commonly given CAM treatment was herbal medicines (Zingiber officinalis, Curcuma longa, Phyllanthus emblica, Azadirachta indica, Syzygium aromaticum, Ocimum sanctum and Allium sativum) followed by Ayurveda medicines such as Brahmi churnam, Rajanyadi churnam, Triphaladi churnam, Ashta churnam and Hinguvachadi churnam. All the CAM therapy was taken orally and no external or parenteral use was noticed. Parents/ caregivers tried CAM therapy since the core problems of the ASD did not improve with the existing therapy and expected

Rajanandh Muhasaparur Ganesan

mgrpharm@gmail.com

1 Department of Pediatrics, Sri Ramachandra Medical College and Research Institute, Deemed to be University, Porur, Chennai, Tamil Nadu, India

2 Department of Pharmacy Practice, Faculty of Pharmacy, Sri Ramachandra Institute of Higher Education and Research, Deemed to be University, Porur, Chennai, Tamil Nadu 600 116, India
CAM therapy to suppress the progression atleast. No adverse effects are reported by any of the CAM treatments. No statistically significant difference exists between the usage of CAM and baseline characters except gender $(p<0.008)$. Among the parents/caregivers of CAM given children, $56.1 \%$ of the parents/caregivers did not discuss about the usage of CAM to their child with the pediatrician. The usage of CAM therapy in ASD children was less when compared to other studies. Herbals and Ayurveda were frequently given CAM to ASD children. History of CAM therapy usage should be a part of medication history interview.

\section{Compliance with Ethical Standards}

Conflict of Interest None.

\section{References}

1. Lee J, Lee SH, Lee B, Yang IJ, Chang GT. A survey of Korean medicine doctors' clinical practice patterns for autism spectrum disorder: preliminary research for clinical practice guidelines. BMC Complement Altern Med. 2018;18:90.

2. Höfer J, Hoffmann F, Kamp-Becker I, et al. Complementary and alternative medicine use in adults with autism spectrum disorder in Germany: results from a multi-center survey. BMC Psychiatry. 2019;19:53.

3. Lindly O, Thorburn S, Heisler K, Reyes N, Zuckerman K. Parent disclosure of complementary health approaches used for children with autism spectrum disorder: barriers and facilitators. Complement Ther Med. 2017;35:47-52.

Publisher's Note Springer Nature remains neutral with regard to jurisdictional claims in published maps and institutional affiliations. 